\title{
Localisation of Inspection Probes in A Storage Tank
}

\author{
Nurmi Elisya Rosli ${ }^{1 *}$, Ali Sophian ${ }^{1}$, Arselan Ashraf ${ }^{2}$ \\ ${ }^{1}$ Department of Mechatronics Engineering, Kulliyah of Engineering, International Islamic University of Malaysia, Malaysia \\ ${ }^{2}$ Department of Electrical and Computer Engineering, Kulliyyah of Engineering, International Islamic University of Malaysia, Malaysia
}

\begin{abstract}
Indoor Positioning System (IPS) has been widely used in today's industry for the various purposes of locating people or objects such as inspection, navigation, and security. Many research works have been done to develop the system by using wireless technology such as Bluetooth and Wi-Fi. The techniques that can give some better performances in terms of accuracy have been investigated and developed. In this paper, ZigBee IEEE 802.15.4 wireless communication protocols are used to implement an indoor localization application system. The research is focusing more on analyzing the behaviour of Received Signal Strength Indicator (RSSI) reading under several conditions and locations by applying the Trilateration algorithm for localizing. The conditions are increasing the number of transmitters, experimented in the non-wireless connection room and wireless connection room by comparing the variation of RSSI values. Analysis of the result shows that the accuracy of the system was improved as the number of transmitters was increased.
\end{abstract}

This is an open access article under the CC BY-SA license

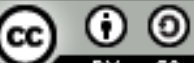

\author{
Keywords: \\ Indoor Positioning System (IPS); \\ Received Signal Strength Indicator \\ (RSSI); \\ Trilateration:
}

Article History:

Received: October 31, 2021

Revised: November 8, 2021

Accepted: November 11, 2021

Published: November 28, 2021

\section{Corresponding Author:}

Nurmi Elisya Rosli

Department of Mechatronics

Engineering, Kulliyah of

Engineering, International Islamic

University of Malaysia, Malaysia

Email: elisya96@gmail.com

\section{INTRODUCTION}

Storage tanks are containers made of steel and function to hold liquids, compressed gases or mediums, that can be hot or cold, for the short- or long-term. They can degrade due to the external environment, such as oil, chemical, and petroleum, leading to corrosion, crack, and leakage to the tank's surface. In 1987, South Dakota School was shut down due to leakage from the bottom of the aboveground storage tank [1][2]. This incident jeopardises the people and environmental well-being. This is supported by [3, 4, 5], which say these damages become threats to human safety, business loss and environmental pollution. Inspection for the storage tank must be done periodically to prevent it from worsening. The process required a specific procedure and setup every time the inspection needed to be done. It needs to start screening from the beginning and does not have any history on past data to analyse the corrosion, crack, and leakage location. The latest technology proposed to improve the current approach for tank inspection purposes by appending an indoor positioning system on the inspection probes.

This paper proposed integrating the indoor localising application system with the probe sensor using the RSSI value from ZigBee wireless communication. A ZigBee, indoor personnel positioning system, has been designed using the Trilateration algorithm and Threshold noise filtering to reduce the error caused by the signal interference and improve the positioning precisely. This paper mainly designed an indoor positioning system using ZigBee technology and discussed the system's performance by varying several parameters and variables.

\section{MATERIAL AND METHOD}

Wireless Technology

Bluetooth Low Energy (BLE) has widely used in today technologies. iBeacon sensor used Bluetooth signal to transmit data to mobile devices. Thus, coordinating and positioning can be obtained through this communication with the range of $30 \mathrm{~m}$ and required to install several 
beacons at a fixed position for a wide range area. The server does data saving and mapping while the Beacon device is installed for a particular distance. The person with the mobile device has a transmit-receive signal with the nearest Beacon. The Mapping table of beacons and locations is stored in the system server to map the real locations. However, this method cannot be used to determine the exact coordinate or location of the user since the precision may be affected by the transmission signal and other environmental factors [6].

$\mathrm{WiFi}$ technology has the highest operation range for indoor positioning purposes. The proposed mechanisms for this system use an access point (AP) with multiple antennas as nearby anchors. The person/things needed to be located will receive-transmit signal communication with WiFi through mobile platforms. Many researchers use several techniques to locate the target's coordinate. Firstly, use ToA, which is the Time of arrival. It is the travel time between a transmitter and a receiver. It needs at least three transmitters to have a plane domain (2 dimensions) localisation. Its localising performance depends on the signal's bandwidth as well as the sampling rate. Next is the Angle of Arrival (AoA) measurement technique where it determines the incoming signal directly from a transmitter on the antenna array.

This approach requires two transmitters with antenna arrays at different places to obtain an object position. Hybrid of AoA/ToA is introduced due to the complicated indoor environment and a limited number of the nearby transmitter by using the data measured from AoA and ToA. Lastly, these approaches are widely used in the industry: Received Signal Strength (RSS) Fingerprint. RSS- alone obtained the distance information from the signal strength ratio). However, due to low precision for location, a combination of fingerprint and RSS was introduced. By associating the signal fingerprint with the target, the transmitter can deduce possible locations from a pre-measured fingerprint database, and this requires only one transmitter. However, the signal fingerprint in each grid needs to be measured in advance. The fingerprint database can choose the most similar fingerprint when the object sends the signal toward a nearby transmitter and do the localisation [7][8].

The next technology is using ZigBee communication protocols. Using ZigBee technology, the indoor positioning system obtains RSSI (Received Signal Strength Indication) values to calculate the distances from point to point from the empirical formula. These values then are used to determine the location of the positioning point. RSSI based indoor positioning system can be used for other technologies as well, such as Bluetooth and WiFi. However, there be a significant error in the positioning result due to the signal intensity of the technologies. Furthermore, the error is easy to be interrupted by the external environment.

Consequently, this results in low accuracy of the positioning coordinates. The system is designed by adding an inertial system to improve the positioning accuracy of the ZigBee IPS [9]. The inertial system is used to suppress the random jitter of the RSSI. Furthermore, it can check the motion information of the object/ person and constrain the calculation result of the RSSI. The Inertial system in this research allows the Kalman Filter to reduce the noise and improve the positioning system performance [10]. Combining the ZigBee and inertial system can reduce the noise, where the inertial system is being utilised for short-term stability to weaken the error from the ZigBee and used a step size algorithm to eradicate the error. The cumulative error of the inertial system further improves the positioning effect of the indoor positioning system.

\section{Localisation Algorithm}

Trilateration is one of RSS based indoor positioning approaches to estimate the position coordinates of the coordinator node. The positioning can be done by calculating the distance between at least three static/known points with the coordinator nodes [11]. As in Figure 1, the concept of trilateration is RSS based, where the value of signal strength is used to calculate 
distance and obtain the position. Triangulation is based on the geometric properties of triangles by measuring angles to determine location. This positioning method forms a circle centred at the access point. The radius of each circle is determined by measuring the signal strength of the moving terminal. In practice, it is almost impossible to obtain a single interaction point due to errors in measurements. In addition, the obstacles and flawed propagation models used may affect signal strength measurements.

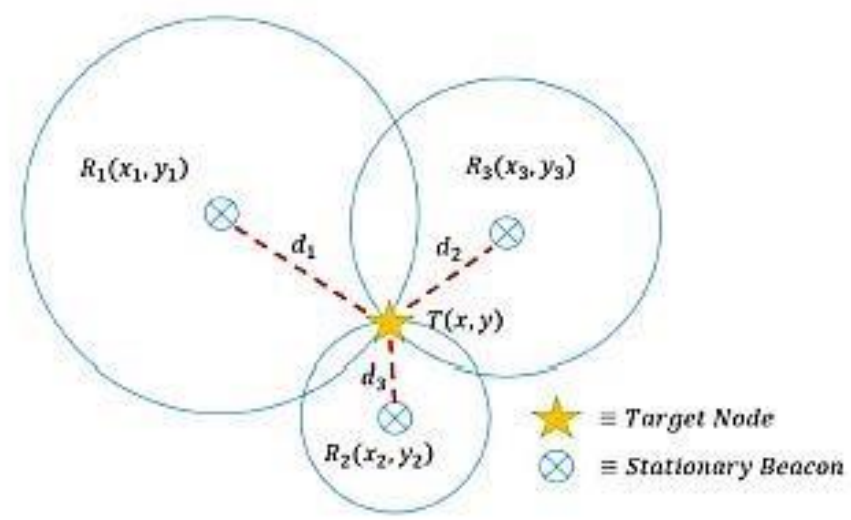

Figure 1. Trilateration algorithm

Four popular techniques have been evolved based on the triangulation method, which is Time of Arrival (TOA), Time Difference of Arrival (TDOA), Angle of Arrival (AOA) and Received Signal Strength (RSS). RSSI in the triangulation method utilises the characteristic of radio propagation over space.

The following technique is multirateration, where this algorithm is a surveillance technique based on the measurement of the difference in distance between two points at known locations by transmitting the signals at known times. The infinite number of locations satisfies the measurement obtained from an absolute distance or angle measurements and the distance between two points. When these possible locations are plotted, they form a hyperbolic curve. To obtain the exact location along that curve, it relies upon multiple measurements obtained from the second measurement taken from a different pair of points, which produces a second curve. This second curve intersects with the first one. When these two curves intersect, it reduces the number of possible locations to improve the system's performance [12].

A typical RSS-based technique is applied to make the detection device in the room is fingerprinting pattern recognition. The pattern matches with a database of signal patterns collected previously. This technique needs to obtain the previous data collection pattern or an offline phase to build the database while the phase detection or pattern matching with an online database called the phase pattern.

This algorithm collects features from the environment that has better localisation performance compared to the previous algorithm discussed. The features collected are commonly based on signal analysis like Received Signal Strength (RSS). However, the drawback of fingerprinting is that the process must redo the offline phase because there might be changes in RSS reception as the interior layout of the room change and the change of access points and other positions.

\section{Noise Filtering}

The drawback of RSS-based detection is the receiving data might fluctuate due to several factors such as electromagnetic noise, physical barriers, and variations in device specifications $[13,14,15]$, which results in inaccuracy. To solve this problem, it needs filter algorithms so 
that the received data becomes accurate. From the study of previous work, the positioning system of RSS based can be improved by applying Kalman Filter. It is an algorithm used in data fusion for a linear system. There might be more than one measuring device on the same parameters for the prediction and correction process to use this filter. It has long-term stability of the RSSI positioning and the short-term stability of the inertial system to complete the data fusion. From the data and information obtained regarding the access point and moving terminal, the coordinates of the person/object can be calculated accurately if the step size of the person is known. However, the step size of the person is changing within a specific range, and the Kalman filter is used to follow the change of step size effectively and continuously adjust the Kalman gain to reduce the interference of this noise [9].

However, Extended Kalman Filter (EKF) can be used for a non-linear object and is widely used in the algorithm for estimation due to its simplicity and good estimation result. According to [8], the EKF is used to create an offline mapping from the environment by inferring the surrounding objects' radar cross-section (RCS). However, the result obtained from a sensitive sensor may have noise and error, which is a disturbance for the mapping. Applying the EKF can help overcome this problem since it is more practical and less complicated [10].

Next is the Trimmed mean filter and also known as a truncated filter. In this method, firstly, the data need to be put in order from smallest to largest and eliminate the selected or outlier data under a specific range from each end of the ordered list. Then, the mean of the remaining values must be calculated. In localisation, when more than necessary distance measurements are required, the location is found as the arithmetic average of the estimates obtained using all three combinations of distance measurements [16, 17, 18]. Thus, applying this filter can increase the performance and accuracy of the system by deleting the outlier and averaging the rest of the values.

\section{Designing the Localisation System}

In this research, the setup consists of $1 \mathrm{ZigBee}$ module as coordinator, which acts as a signal receiver, and three ZigBee modules as Endpoint, E1, E2 and E3 that transmit the signal for wireless communication. The coordinator is attached to a mobile robot for tracking purposes. The endpoints will have fixed in known coordinates in the field site. The RSSI values obtained by the coordinator will be used to calculate the mobile robot's position based on the trilateration algorithm, as displayed in Figure 2.

\section{Project Development Location \& Setup}

The field of the project was developed at Project Development Lab at Kulliyah of Engineering, E5. Since the project will be applied for a storage tank, it can be said that most of the storage tank in the industry nowadays is in the shape of a circular cylinder. The circle wall is made from the model board with a $1.5 \mathrm{~m}$ since that is the best operating range for wireless communication between the ZigBee. The circumference of the circle is $4.71 \mathrm{~m}$, with an area of $1.767 \mathrm{~m} 2$. The position of each endpoint has been determined by dividing the circumference into 3, whereby the position is shown in Figure 3. 


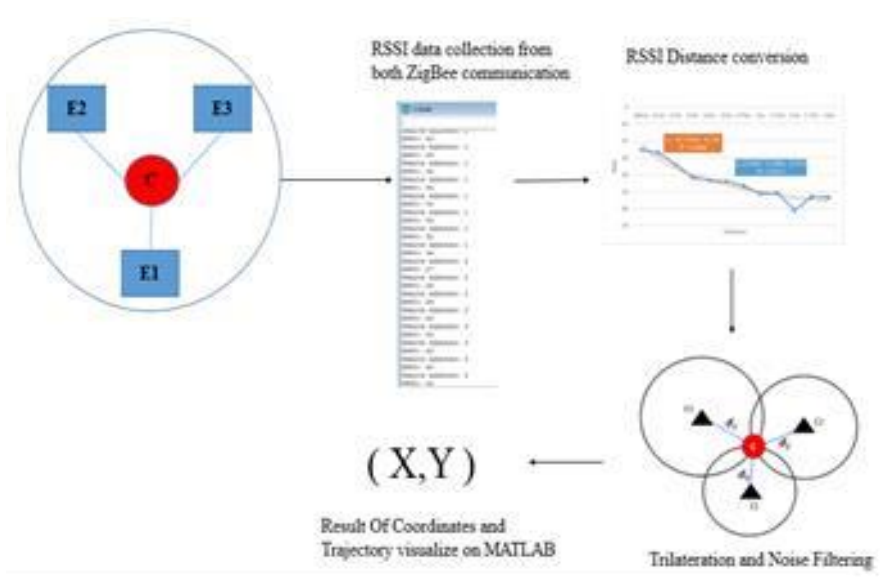

Figure 2. Overall of the system design

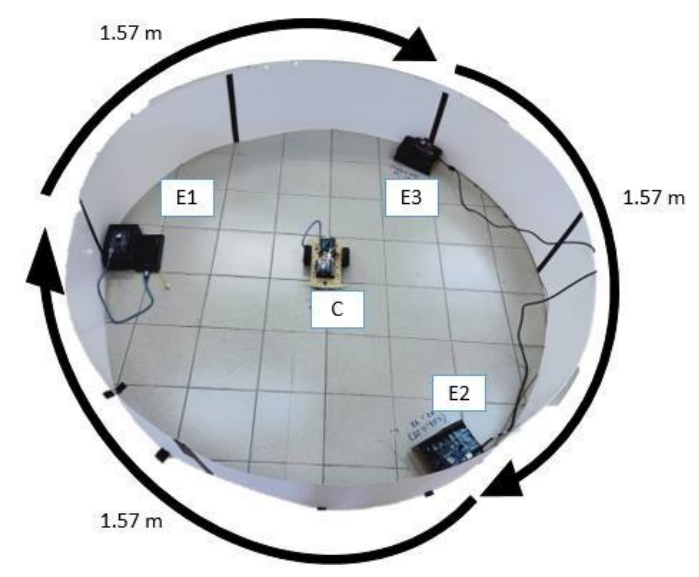

Figure 3. Site Field of the project

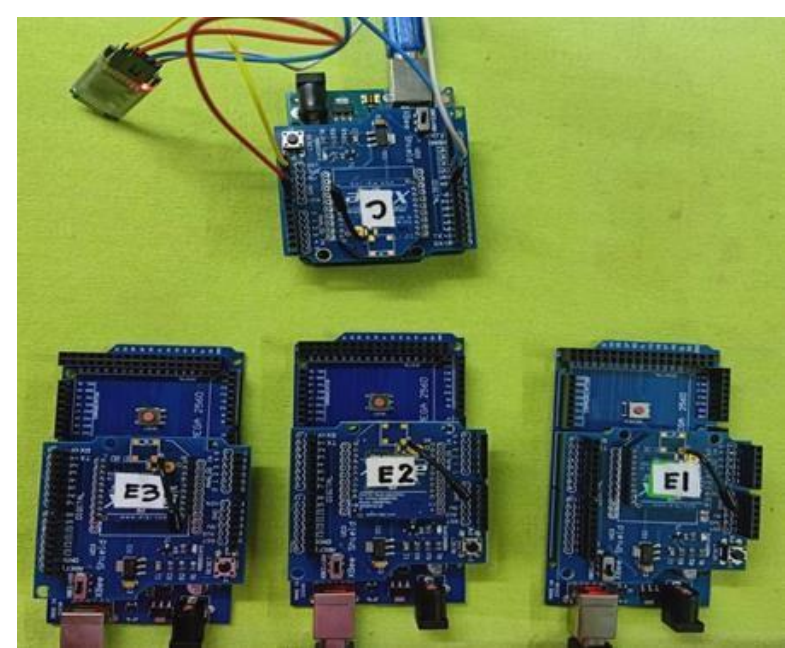

Figure 4. The Connection of The Zigbee Coordinator and Endpoint

\section{ZigBee Communication Networking}

For ZigBee communication, ZigBee Series 1 is used for both coordinator and endpoint, where it is interfaced to ZigBee Shield on Arduino Uno as in Figure 4. The coordinator receives the signal strength from the three endpoints and processes the RSSI reading in Arduino to generate distance for each endpoint from the mobile car. 


\section{Distance Calibration}

From the ZigBee communication networking, the RSSI value received by the coordinator will be used to calculate the distance of the endpoint to the mobile robot. An experiment is conducted to obtain the RSSI values by varying the distances for each endpoint. The output of the experiment was used to calibrate and generate the formula conversion from RSSI to distance. The distance calculated then be used for positioning. Equation (1) has been established from the calibration.

$$
\begin{aligned}
& \mathrm{d} 1=\sqrt[z]{\frac{(\text { RSSI } 1+54.806)}{0.264}}+\mathbf{1 1 . 8 1 5 3 4} \\
& \mathrm{d} 2=\sqrt[2]{\frac{(\text { RSSI } 2+24.0599)}{0.2461}}+2.52154 \\
& \mathrm{~d} 3=\sqrt[2]{\frac{(\text { RSSI } 2+56.01704)}{0.2217}}+12.4862
\end{aligned}
$$

RSSI is the signal received by the coordinator from each endpoint. $\mathrm{d} 1, \mathrm{~d} 2$ and $\mathrm{d} 3$ is the distance calculated between the coordinator on the mobile car to the endpoint.

\section{Trilateration in $2 D$ algorithm}

In this project, 2D Trilateration is used to locate the coordinates of the mobile robot, which be on the XY plane. The principle of this method is to compute the intersection of three known radii and the center of a coordinate circle, as shown in Figure 5.

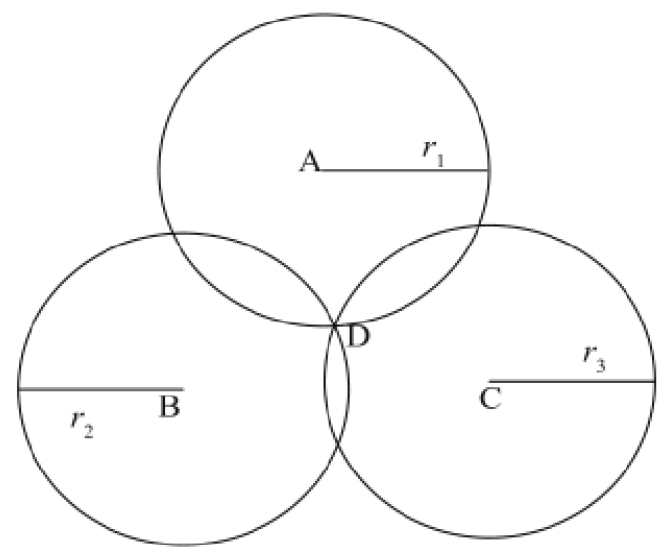

Figure 5. Trilateration localisation principle

The positions of three ZigBee endpoints are known in Table 1 for the algorithm to work accordingly.

\begin{tabular}{ccc}
\multicolumn{3}{c}{ Table 1. Known Coordinates for The Three Endpoint } \\
\hline Position E1 & Position E2 & Position E3 \\
\hline$(0,-0.70)$ & $(0.70,0.25)$ & $(-0.70,-0.25)$ \\
\hline
\end{tabular}

Once the distance from the three endpoints is generated, the data is filtered using running average and threshold noise filtering to increase the accuracy of the positioning coordinates later. Then, the Trilateration algorithm is applied to find the coordinates of the mobile robot. The Trilateration algorithm is as shown in (2). 


$$
\begin{aligned}
& \left(x-x_{1}\right)^{2}+\left(y-y_{1}\right)^{2}=d_{1}^{2} \\
& \left(x-x_{2}\right)^{2}+\left(y-y_{2}\right)^{2}=d_{2}^{2} \\
& \left(x-x_{3}\right)^{2}+\left(y-y_{3}\right)^{2}=d_{3}^{2}
\end{aligned}
$$

Where:

$$
\begin{aligned}
& A x+B y=C \\
& D x+E y=F
\end{aligned}
$$

Then

$$
\begin{aligned}
& A=-2 x_{1}+2 x_{2} \\
& B=-2 y 1+2 y 2 \\
& C=d 12-d 22-x 12+x 22-y 12+y 22 \\
& D=-2 x 2+2 x 3 \\
& E=-2 y_{2}+2 y_{3} \\
& F=d_{2}^{2}-d_{3}^{2}-x_{2}^{2}+x_{3}^{2}-y_{2}^{2}+y_{3}^{2}
\end{aligned}
$$

To obtain the coordinates for the $\mathrm{x}$ and $\mathrm{y}$ plane, (5) has been used:

$$
\begin{aligned}
& x=\frac{C E-F B}{E A-B D} \\
& y=\frac{C D-A F}{B D-A E}
\end{aligned}
$$

\section{Noise Filtering}

Before applying the trilateration algorithm, the data of the distance obtained for each point be filtered by applying a trimmed mean filter and calculating the average of every ten distance samples. The trimmed mean is a method of averaging that removes any outliers or a small percentage of the highest and lowest values before calculating the means. This method has been proven in the experiment conducted to observe the difference when applying the filter to the system as in Figure 6. The formula used shows in (6).

$$
\mu=\frac{\sqrt{X_{i}}}{n}
$$

$\mathrm{Xi}$ is the trimmed distance set sum, while $\mathrm{n}$ is the total numbers trimmed distance set and $\mu$ is the trimmed mean. 


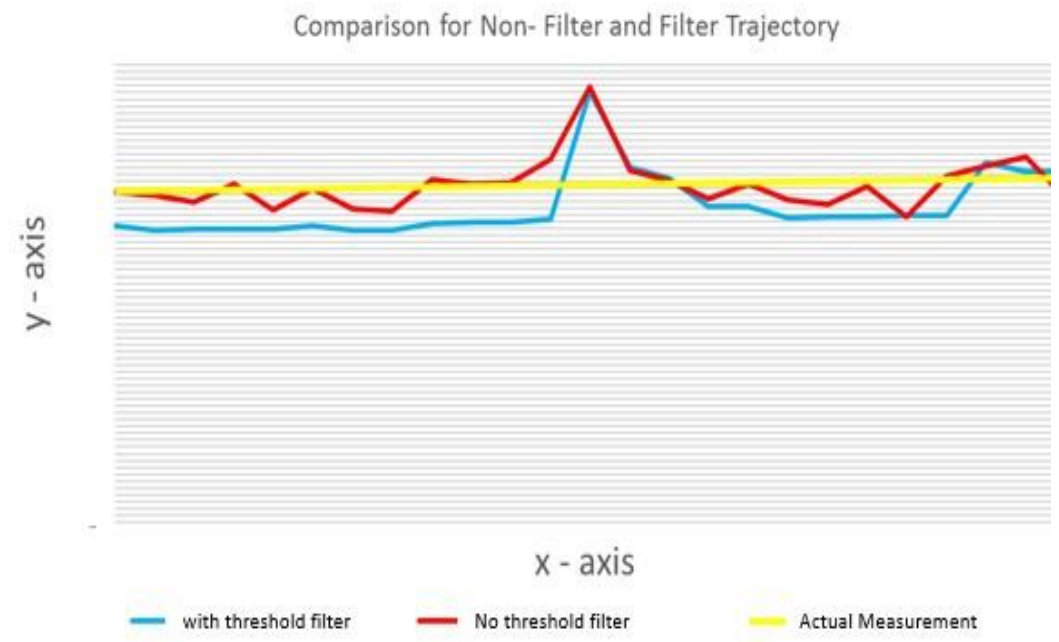

Figure 6. Comparison of trajectory for non-filter and filtered localization

\section{RESULTS AND DISCUSSION}

After expanding and performing the mathematical operation in (2), the equation then is simplified as the experiment conducted is to compare (3) and (4) accuracy of the coordinates as the number of the transmitter being increased from (3) to (4) using the same algorithm and noise filtering as tabulated in Table 2 and Table 3. For three endpoints, from the trajectory in Figure 7, it can be observed that the trajectory follows the mobile robot's horizontal movement and is plotted at the negative side of the $\mathrm{x}$ - plane. However, at origin $(0,0)$, there was an impactful change in the value of y coordinates.

Table 2. Positioning Result for 3 Endpoint

\begin{tabular}{|c|c|c|c|c|}
\hline $\mathbf{X}$ & $\mathbf{Y}$ & $\begin{array}{c}\mathrm{X} \\
\text { experiment }\end{array}$ & $\begin{array}{c}\mathrm{Y} \\
\text { experiment }\end{array}$ & $\begin{array}{c}\text { Error } \\
(\%)\end{array}$ \\
\hline 0 & 0 & 0.16 & -1.07 & 0 \\
\hline 0.05 & 0 & -0.4 & 0.19 & -140 \\
\hline-0.05 & 0 & -0.78 & 0.18 & -178 \\
\hline 0.1 & 0 & -0.48 & 0.19 & -148 \\
\hline-0.1 & 0 & -0.8 & 0.18 & -180 \\
\hline 0.15 & 0 & -0.69 & 0.19 & -169 \\
\hline-0.15 & 0 & -0.8 & 0.18 & -180 \\
\hline 0.2 & 0 & -0.69 & 0.18 & -169 \\
\hline-0.2 & 0 & -0.81 & 0.18 & -181 \\
\hline 0.25 & 0 & -0.77 & 0.18 & -177 \\
\hline-0.25 & 0 & -0.86 & 0.19 & -186 \\
\hline 0.3 & 0 & -0.76 & 0.18 & -176 \\
\hline-0.3 & 0 & -0.86 & 0.19 & -186 \\
\hline 0.35 & 0 & -0.76 & 0.17 & -176 \\
\hline-0.35 & 0 & -0.83 & 0.19 & -183 \\
\hline 0.4 & 0 & -0.75 & 0.17 & -175 \\
\hline-0.4 & 0 & -0.85 & 0.19 & -185 \\
\hline 0.45 & 0 & -0.75 & 0.17 & -175 \\
\hline-0.45 & 0 & -0.85 & 0.19 & -185 \\
\hline 0.5 & 0 & -0.36 & 0.18 & -136 \\
\hline-0.5 & 0 & -0.85 & 0.19 & -185 \\
\hline 0.55 & 0 & -0.43 & 0.18 & -143 \\
\hline-0.55 & 0 & -0.86 & 0.19 & -186 \\
\hline 0.6 & 0 & -0.43 & 0.18 & -143 \\
\hline-0.6 & 0 & -0.83 & 0.19 & -183 \\
\hline
\end{tabular}


Table 3. Positioning Result for 4 Endpoint

\begin{tabular}{|c|c|c|c|c|}
\hline $\mathbf{X}$ & $\mathbf{Y}$ & $\begin{array}{c}\mathrm{X} \\
\text { experiment }\end{array}$ & $\begin{array}{c}\mathrm{Y} \\
\text { experiment }\end{array}$ & $\begin{array}{c}\text { Error } \\
(\%) \\
\end{array}$ \\
\hline 0 & 0 & -1.06 & -0.9 & \\
\hline 0.05 & 0 & -0.12 & -2.15 & -340 \\
\hline-0.05 & 0 & -0.13 & -2.18 & 160 \\
\hline 0.1 & 0 & -0.14 & -2.22 & -240 \\
\hline-0.1 & 0 & -0.12 & -2.18 & 20 \\
\hline 0.15 & 0 & -0.15 & -2.23 & -200 \\
\hline-0.15 & 0 & -0.13 & -2.2 & -13.3333 \\
\hline 0.2 & 0 & -0.14 & -2.21 & -170 \\
\hline-0.2 & 0 & -0.14 & -2.22 & -30 \\
\hline 0.25 & 0 & -0.13 & -2.19 & -152 \\
\hline-0.25 & 0 & -0.15 & -2.24 & -40 \\
\hline 0.3 & 0 & -0.14 & -2.2 & -146.667 \\
\hline-0.3 & 0 & -0.15 & -2.23 & -50 \\
\hline 0.35 & 0 & -0.14 & -2.2 & -140 \\
\hline-0.35 & 0 & -0.14 & -2.22 & -60 \\
\hline 0.4 & 0 & -0.13 & -2.19 & -132.5 \\
\hline-0.4 & 0 & 0.14 & -2.21 & -135 \\
\hline 0.45 & 0 & -0.14 & -2.2 & -131.111 \\
\hline-0.45 & 0 & -0.15 & -2.23 & -66.6667 \\
\hline 0.5 & 0 & -0.14 & -2.21 & -128 \\
\hline-0.5 & 0 & -0.14 & -2.22 & -72 \\
\hline 0.55 & 0 & -0.14 & -2.21 & -125.455 \\
\hline-0.55 & 0 & -0.15 & -2.22 & -72.7273 \\
\hline 0.6 & 0 & -0.13 & -2.22 & -121.667 \\
\hline-0.6 & 0 & 8.74 & -1.04 & -1556.67 \\
\hline
\end{tabular}

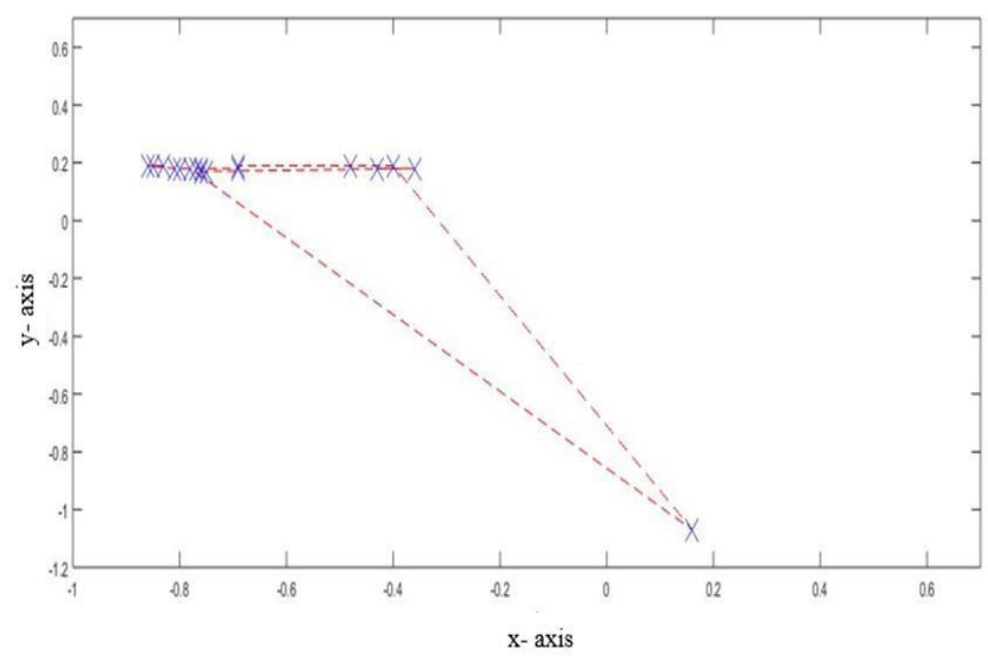

Figure 7. Left to right motion of the mobile robot using three endpoints

For using four endpoints, as seen from Figure 8, the mobile robot's trajectory is plotted smoothly compared to 3 endpoints. Based on the analysis of the results and trajectory, it can be concluded that using four transmitters has higher accuracy than three transmitters, which shows that the system's accuracy increases as the number of transmitters increases. This is because some of the coordinates obtained were very far from the actual coordinates. Thus, it can be concluded that the positioning system developed in this project has low accuracy. 


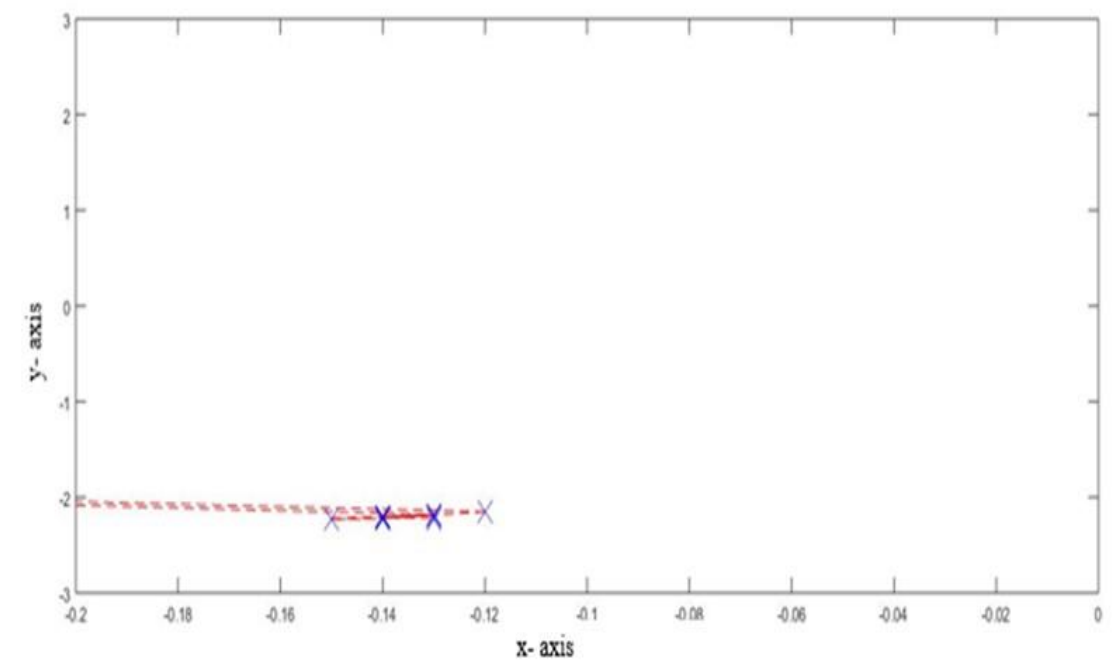

Figure 8. Left to right motion of the mobile robot using four endpoints

Next, another experiment was conducted to compare the coordinates when applying filter and no- filter algorithm using three endpoints and tabulated in Table 4. From the trajectory visualise in Figure 9 and Figure 10, it can be observed that the trajectory from the left to right motion of the mobile car are much smoother when the truncated filter is applied.

Table 4. Comparison for Positioning Result of 3 Endpoint with and Without Filter

\begin{tabular}{clcccc}
\hline $\mathbf{X}$ & $\mathbf{Y}$ & $\begin{array}{c}\mathbf{X} \\
\text { measured } \\
\text { with } \\
\text { filter }\end{array}$ & $\begin{array}{c}\mathbf{Y} \\
\text { measured } \\
\text { with } \\
\text { filter }\end{array}$ & $\begin{array}{c}\mathbf{X} \\
\text { measured } \\
\text { without } \\
\text { filter }\end{array}$ & $\begin{array}{c}\mathbf{Y} \\
\text { measured } \\
\text { without } \\
\text { filter }\end{array}$ \\
\hline 0.05 & 0 & -0.16 & -1.27 & -0.1 & -1.08 \\
-0.05 & 0 & -0.4 & 0.19 & -0.42 & 0.18 \\
0.1 & 0 & -0.78 & 0.18 & -0.34 & 0.21 \\
-0.1 & 0 & -0.48 & 0.19 & -0.5 & 0.18 \\
0.15 & 0 & -0.8 & 0.18 & -0.51 & 0.18 \\
-0.15 & 0 & -0.69 & 0.19 & -0.63 & 0.17 \\
0.2 & 0 & -0.8 & 0.18 & -0.52 & 0.19 \\
-0.2 & 0 & -0.69 & 0.18 & -0.52 & 0.17 \\
0.25 & 0 & -0.81 & 0.18 & -0.49 & 0.18 \\
-0.25 & 0 & -0.77 & 0.18 & -0.64 & 0.16 \\
0.3 & 0 & -0.86 & 0.19 & -0.72 & 0.18 \\
-0.3 & 0 & -0.76 & 0.18 & -0.67 & 0.17 \\
0.35 & 0 & -0.86 & 0.19 & -0.7 & 0.18 \\
-0.35 & 0 & -0.76 & 0.17 & -0.54 & 0.17 \\
0.4 & 0 & -0.83 & 0.19 & -0.55 & 0.2 \\
-0.4 & 0 & -0.75 & 0.17 & -0.76 & 0.17 \\
0.45 & 0 & -0.85 & 0.19 & -0.71 & 0.19 \\
-0.45 & 0 & -0.75 & 0.17 & -0.46 & 0.16 \\
0.5 & 0 & -0.85 & 0.19 & -0.52 & 0.19 \\
-0.5 & 0 & -0.36 & 0.18 & -0.39 & 0.17 \\
0.55 & 0 & -0.85 & 0.19 & -0.65 & 0.2 \\
-0.55 & 0 & -0.43 & 0.18 & -0.32 & 0.16 \\
0.6 & 0 & -0.86 & 0.19 & -0.6 & 0.2 \\
-0.6 & 0 & -0.43 & 0.18 & -0.62 & 0.16 \\
0.05 & 0 & -0.83 & 0.19 & -0.58 & 0.19 \\
\hline & & & & &
\end{tabular}




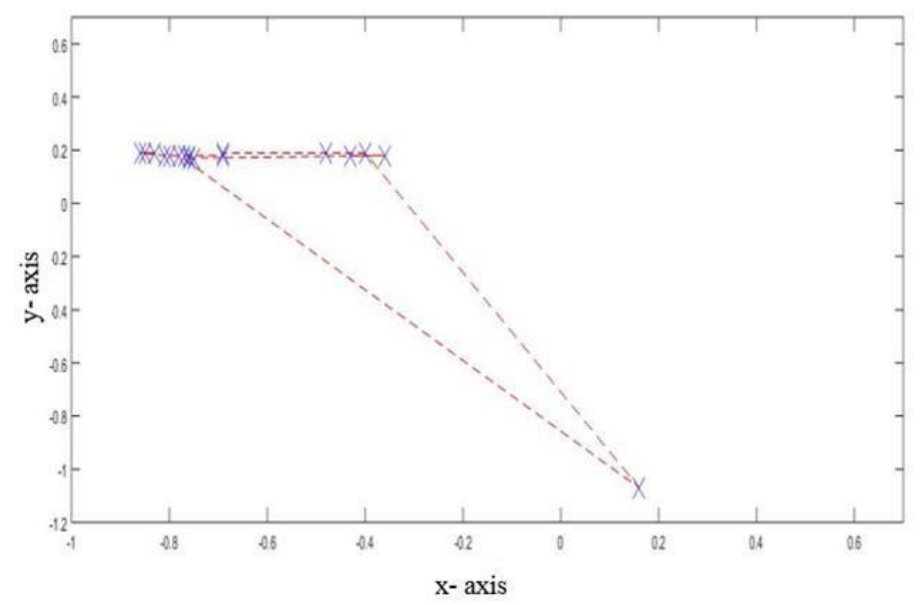

Figure 9. Left to right motion of the mobile robot with a Threshold filter

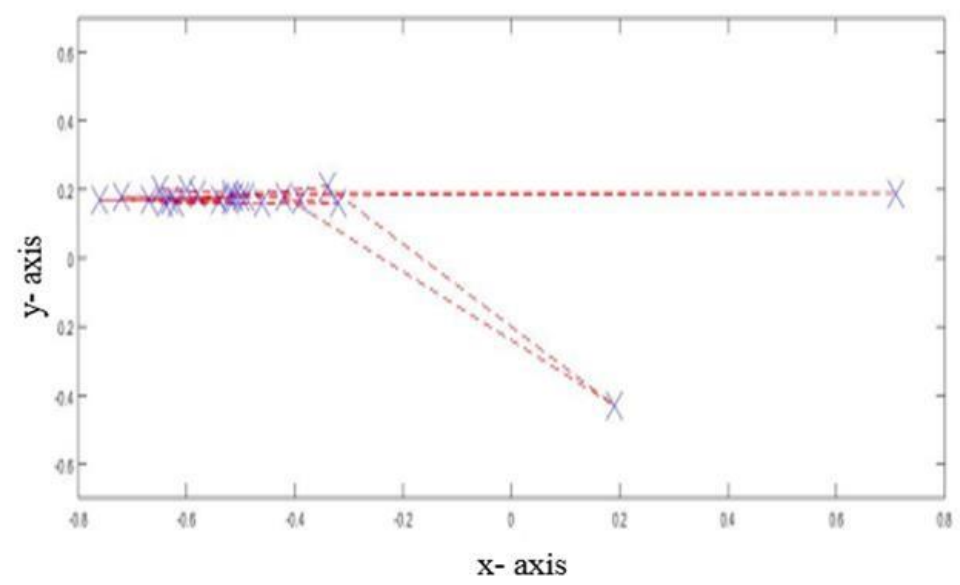

Figure 10. Left to right motion of the mobile robot without Threshold filter

\section{CONCLUSION}

This project proposed an RSS- based indoor positioning system using trilateration on ZigBee wireless data fusion. This project aims to investigate potential indoor positioning techniques cost-effective for industrial users and build a prototype for positioning by using wireless communication. The second objective of this project is to build the prototype of the indoor positioning system and evaluate the performance by observing the accuracy of the approaches applied. These objectives are achieved by conducting experimental setups. Experimental result demonstrates the performance of the positioning system and a possible factor that might influence the performance of the system. It is proven that ZigBee's signal is easily interfered with by the surrounding environment. The interfered signal results in an inaccurate distance calculated from the RSSI value and the calculated position is consequently affected. In implementing this project, there are some limitations where the system proposed can only be applied in a small range of regions since the ZigBee can only operate in a short range of distance. Besides, the noises from the environment surrounding may vary the result of the coordinates. For improvement, there are some recommendations for this project. Firstly, to improve the performance of the indoor positioning system, it is suggested to use higher technology of wireless network which can scale up the region of operation as well. Besides, adding tracking sensors such as Inertial Measurement Unit (IMU) to the system may help to enhance the accuracy of positioning outcomes. Next, the system can apply the Kalman Filter to reduce the possible noise that might affect the localisation system. 


\section{REFERENCES}

[1] T. U. Symposium., "Catastrophic Tank Failures: Highlights of Past Failures along with Proactive Tanks Designs." Catastrophic Tank Failures: Highlights of Past Failures along with Proactive Tanks Designs, US, 2002.

[2] Y. Yea, J. Lua, J. Ding, W. Wang, and J. Yan, "Numerical simulation on the storage performance of a phase change materials based metal hydride hydrogen storage tank," Applied Energy, vol. 278, 115682, 2020, doi: 10.1016/j.apenergy.2020.115682

[3] R. Anvo, T. P. Sattar, T. Gan and I. Pinson, "Non-destructive Testing Robots (NDTBOTs) for In-Service Storage Tank Inspection," Journal of Mechanics Engineering and Automation, vol. 8, no. 3, pp. 103-109, 2018, doi: 10.17265/2159-5275/2018.03.001

[4] M. Gwaindepi and T. Mushiri, "Enhancing In-Service Tank Maintenance Through Industrial Internet of Things: A Case for Acoustic Emission Tank Inspection," in Innovations in the Industrial Internet of Things (IIoT) and Smart Factory, IGI Global: US, 2021, doi: 10.4018/978-1-7998-3375-8.ch017

[5] J. Pan and S. Liang, "A study on the buckling behavior of in-service large open-topped oil-storage tanks," Structures, vol. 29, pp. 211-224, 2021, doi: 10.1016/j.istruc.2020.11.034

[6] X. Lin, T. Ho, C. Fang, Z. Yen, B. Yang and F. Lai, "A mobile indoor positioning system based on iBeacon technology," 2015 37th Annual International Conference of the IEEE Engineering in Medicine and Biology Society (EMBC), 2015, pp. 4970-4973, doi: 10.1109/EMBC.2015.7319507.

[7] C. Yang and H. Shao, "WiFi-based indoor positioning," in IEEE Communications Magazine, vol. 53, no. 3, pp. 150-157, March 2015, doi: 10.1109/MCOM.2015.7060497.

[8] L. M. Silalahi, S. Budiyanto, F. A. Silaban, and A. R. Hakim, "Design a Monitoring and Control in Irrigation Systems using Arduino Wemos with the Internet of Things," Journal of Integrated and Advanced Engineering (JIAE), vol. 1, no. 1, pp. 53-64, 2021, doi: 10.51662/jiae.v1i1.13

[9] C. Han, W. Zhongtao and W. Longxu, "Indoor Positioning System Based on Zigbee and Inertial System," 2018 5th International Conference on Dependable Systems and Their Applications (DSA), 2018, pp. 80-85, doi: 10.1109/DSA.2018.00023.

[10] I. Toroslu and M. Dogan, "Effective Sensor Fusion of a Mobile Robot for SLAM Implementation," 4th International Conference on Control, Automation and Robotics (ICCAR), 2018, doi: 10.1109/ICCAR.2018.8384648

[11] R. T. Haryanto, "Android Smartphone Location Detection on Indoor using Trilateration Method and Kalman Filter," International Journal of Computer Techniques, vol. 5, no. 3, 2018, 30.

[12] A. Guerra et al., "Crowd-Based Personal Radars for Indoor Mapping using UWB measurements," 2016 IEEE International Conference on Ubiquitous Wireless Broadband (ICUWB), 2016, doi: 10.1109/ICUWB.2016.7790437

[13] T. D. Badgaiyan and R Tiwari, "Localisation Algorithm for Multilateration System Using Hyperbolic Localizations," International Journal of Advanced Research in Electronics and Communication Engineering (IJARECE), vol. 7, no. 4, pp. 396-402, 2018

[14] D. W. Upton et al., "A Review of Techniques for RSS-Based Radiometric Partial Discharge Localization," Sensors, vol. 21, no. 3, pp. 909, 2021, doi: 10.3390/s21030909

[15] T. Janssen, R. Berkvens, and M. Weyn, "RSS-Based Localization and Mobility Evaluation Using a Single NB-IoT Cell," Sensors, vol. 20, no, 21, pp. 6172, 2020, doi: 10.3390/s20216172

[16] M. A. Altinkaya, "Alpha-trimmed means of multiple location estimates," 21st Signal Processing and Communications Applications Conference (SIU), Turkey, 2013, doi:10.1109/siu.2013.6531436

[17] N. Goel, H. Kaur and J. Saxena, "Modified decision based unsymmetric adaptive neighborhood trimmed mean filter for removal of very high density salt and pepper noise," Multimedia Tools and Applications, vol. 79, pp. 19739-19768, 2020, doi: 10.1007/s11042-020-08687-y

[18] K. B. Khan, M. Shahid, H. Ullah and M. M. Khan, "Adaptive Trimmed Mean Autoregressive Model for Reduction of Poisson Noise in Scintigraphic Images," IIUM Engineering Journal, vol. 19, no. 2, pp. 68-79, 2018, doi: 10.31436/iiumej.v19i2.835 